Geoscience and Remote Sensing Symposium (IGARSS), 2015 IEEE International. 26-31 July 2015, Milan Italy

\title{
Remote sensing of surface ocean PH exploiting sea surface salinity satellite observations
}

\author{
Sabia Roberto 1, Fernandez-Prieto Diego 2, Shutler Jamie ${ }^{3}$, Donlon Craig ${ }^{4}$, Land Peter 5 , \\ Reul Nicolas 6
}

${ }^{1}$ European Space Agcy, Estec, Telespazio Vega UK Ltd, NL-2200 AG Noordwijk, Netherlands.

2 European Space Agcy, ESRIN, Frascati, Italy.

3 Univ Exeter, Exeter, Devon, England.

${ }^{4}$ European Space Agcy, Estec, NL-2200 AG Noordwijk, Netherlands.

5 Plymouth Marine Lab, Plymouth, Devon, England.

6 IFREMER, Toulon, France.

\begin{abstract}
:
The overall process commonly referred to as Ocean Acidification $(O A)$ is nowadays gathering increasing attention for its profound impact at scientific and socioeconomic level. To date, the majority of the scientific studies into the potential impacts of OA have focused on models and in situ datasets. Satellite remote sensing technology have yet to be fully exploited and could play a significant role by providing synoptic and frequent measurements for investigating OA processes on global scales. Within this context, the purpose of the ESA "Pathfinders-OA" project is to quantitatively and routinely estimate surface ocean $\mathrm{pH}$ by means of satellite observations in several ocean regions. Satellite Ocean Colour, Sea Surface Temperature and Sea Surface Salinity data (with an emphasis on the latter) will be exploited. A proper merging of these different datasets will allow to compute at least two independent proxies among the seawater carbonate system parameters and therefore obtain the best educated guess of the surface ocean $\mathrm{pH}$. Preliminary results of the anomaly and variability of the ocean $\mathrm{pH}$ maps are presented.
\end{abstract}

Keywords : Ocean Acidification, Ocean pH, Sea Surface Salinity, Sea Surface Temperature, Carbon dioxide 
The surface ocean currently absorbs approximately one third of the excess carbon dioxide (CO2) injected into the atmosphere from human fossil fuel burning and deforestation, mitigating the impact of global warming and climate change. However, this anthropogenic CO2 absorption by seawater determines a reduction of both ocean $\mathrm{pH}$ and the concentration of carbonate ion. The latter can also lead to a decrease in calcium carbonate saturation state, with potential implications for marine animals, especially calcifying organisms. The overall process is commonly referred to as Ocean Acidification (OA), and is nowadays gathering increasing attention as one of the major foci of climate-related research [1,2], having profound impact not only at scientific level, but also in its socio-economic dimension. Areas that have shown to be already particularly vulnerable to OA include upwelling regions, the oceans near the poles and coastal regions that receive freshwater discharges.

Growing international efforts are being devoted to develop a coordinated strategy for monitoring OA [3], with an eager need for global and frequent observations of OA-relevant parameters; however, the datasets acquired are currently mostly relevant to in-situ measurements, laboratory-controlled experiments and models simulations. Remote sensing technology can be integrated by providing synoptic and frequent OA-related observations, upscaling and extending in-situ carbonate chemistry measurements on different spatial/temporal scales; yet, the preliminary products developed so far are only regional, empirical or derived with a limited variety of satellite datasets $[4,5]$.

Within this context, "Pathfinders-OA" is an ESA project (led by Plymouth Marine Laboratory, UK) meant to exploit Earth Observation (EO) to monitor ocean acidification. This is being addressed by collecting relevant datasets and creating a large database of EO-in situ matchups, with the objective of developing and validating algorithms to retrieve OA parameters from space in 5 case-study regions (global ocean, Amazon plume, Barents sea, Greater Caribbean, Bay of Bengal). 
Overall, the purpose of this study is to quantitatively and routinely estimate surface ocean $\mathrm{pH}$ by means of satellite observations, capitalizing on the recent advent of remotely-sensed salinity measurements. The overarching objectives are to develop new algorithms and data processing strategies to overcome the relative immaturity of OA satellite products currently available, and to produce a global, temporally evolving, quasi-operational suite of relevant satellite-derived data.

Specifically, this will be performed by exploiting the information content of Ocean Colour (OC) data, Sea Surface Temperature (SST), Wind Speed (WS) and Sea Surface Salinity (SSS) parameters (with an emphasis on the latter). A proper merging of these different satellites datasets will allow to compute at least two independent proxies among the seawater carbon dioxide system parameters: namely, the partial pressure of $\mathrm{CO} 2$ in surface seawater (pCO2); the total Dissolved Inorganic Carbon (DIC) and the total alkalinity (AT). Through the knowledge of these parameters, the final objective is to come up with the currently best educated guess of the surface ocean $\mathrm{pH}$.

A preliminary effort in this sense was the estimation of monthly surface ocean pH maps for 2010 in the North Atlantic, using pCO2 data and computed AT derived from SMOS salinity measurements, with a preliminary estimation of the errors in $\mathrm{pH}$ due to inaccuracies in satellite SSS [6]. Currently, ongoing efforts aim at extending the temporal domain and the geographical analysis, including additional satellite datasets (especially OC-related) and the remaining carbonate system parameters, and performing different permutations (round-robin approach) in view of a systematic ocean $\mathrm{pH}$ sensitivity analysis. An overall validation plan is being devised to verify the consistency, accuracy and robustness of the satellite datasets produced, by quality-controlling the satellite products against in-situ measurements and modelling outputs.

The innovation of this study lies mainly in the effort of unifying fragmented remote sensing studies and generating a novel value-added satellite product: a global and frequent surface ocean $\mathrm{pH}$ "cartography”. This will foster the advancement of the embryonic phase of OA-related remote sensing and will aim at bridging the gap between the satellite and the process studies communities, benefiting from their crossfertilization and feedback.

\section{References}

[1] Doney, S.C., Victoria J. Fabry, Richard A. Feely, and Joan A. Kleypas, Ocean Acidification: The

Other CO2 Problem, Annu. Rev. Mar. Sci. 2009. 1:169-92, DOI: 10.1146/annurev.marine.010908.163834. 
[2] Raven, J., K. Caldeira, H. Elderfield, O. Hoegh-Guldberg, P. Liss, U. Riebesell, J. Shepherd, C. Turley, and A. Watson. Ocean acidification due to increasing atmospheric carbon dioxide. The Royal Society, 2005.

[3] Orr, J.C., K. Caldeira, V. Fabry, J.-P. Gattuso, P. Haugan, P. Lehodey, S. Pantoja, H.-O. Pörtner, U. Riebesell, T. Trull, M. Hood, E. Urban, and W. Broadgate (2009) Research Priorities for Ocean Acidification, report from the Second Symposium on the Ocean in a High-CO2 World, Monaco, October 6-9, 2008, convened by SCOR, UNESCO-IOC, IAEA, and IGBP, 25 pp.

[4] Gledhill, D.K., R. Wanninkhof, and C.M. Eakin. 2009. Observing ocean acidification from space. Oceanography 22(4):48-59

[5] Qingyang Sun, Danling Tang \& Sufen Wang (2012): Remote-sensing observations relevant to ocean acidification, International Journal of Remote Sensing, 33:23, 7542-7558.

[6] Sabia, R., D. Fernández-Prieto, J. Shutler, N. Reul, P. Land and C. Donlon; Preparatory activities to estimate surface ocean $\mathrm{pH}$ from satellite observations; ESA-SOLAS-EGU Earth Observation for OceanAtmosphere Interactions Science 2014, Frascati, Italy, October 28-31, 2014. 